International Journal of Difference Equations (IJDE).

ISSN 0973-6069 Volume 16, Number 1 (2021), pp. 35-46

(C) Research India Publications

https://dx.doi.org/10.37622/IJDE/16.1.2021.35-46

\title{
Control of Ball-Plate System with Observer-Based State-Feedback and Geometric Considerations
}

\author{
Khalid Lefrouni \\ Saoudi Taibi \\ Mohammed V University in Rabat, Morocco.
}

Younes Moubachir

Mohammed V University in Rabat, Morocco. Mohammed V University in Rabat, Morocco.

\begin{abstract}
The ball and plate system is a very unstable and non-linear system. So different studies have been interested in this system to develop new control methods and algorithms. In this article, we propose a state feedback controller based on the Luenberger observer that provides an estimate of the system state. To this end, first, considering the feedback delay in the control loop, we determine the state space model of the Ball-Plate System, and secondly, based on the geometric method and the state feedback control developed in the previous work [7], we synthesized an observer. Finally, we tested the effectiveness of our methodology through simulation.
\end{abstract}

Keywords: Luenberger Observer, Frequency-Domain, Ball-Plate System, State Estimation.

\section{INTRODUCTION}

Like the magnetic levitation system [5] and the ball-Beam system [4], the ball and plate system has complex dynamics due to its instability and nonlinearity, so it has attracted special interest in teaching engineering and research. The main advantages of this type of system are primarily the facility of its implementation and the opportunity for experimental verification of theoretical knowledge in modeling, control, and other engineering disciplines [2].

Several works have been reported in literature. In [6], the ball and plate system uses two magnetic actuators for two degrees of freedom. For this system, a controller of ball position using Lyapunov function is designed. The LQR control method is proposed 
in [8]. In [11] the authors discuss the use of PD position controller and PD angle controller to control the ball and plate system. In [3], a new form of proportional derivative control, called dual PD control, is proposed for controlling the position of the ball. Literature [9] compares two control strategies, the PID controller and the sliding mode controller. In [10], four controller are compared: PID, sliding mode, fuzzy and LQR. However, all these studies have been carried out either on a simplified onedimensional model [13] or by assuming a real-time model [12] that does not take into account the delay introduced by the controller within the control loop.

In order to solve this problem, we propose in this article, the synthesis of an observer to estimate the state of the system taking into consideration the feedback delay. To this end, we will use a state feedback controller [1] on the one hand, and on the other hand a frequential method called geometric approach [7].

The rest of this article is structured as follows. In Section 2, we introduced a state space model describing the dynamics of the ball and plate system taking into account feedback delay. Then, in Section 3, we developed a linear observer to estimate the system state. In Section 4, we verified the results obtained through simulation. Finally, we introduce our conclusions and future work in Section 5.

The control law developed in this article is based on a linear model of the ball and plate system that was found by applying Lagrange's method, the following section illustrates the steps followed.

\section{BALL AND PLATE DYNAMICS}

The control law developed in this article is based on a linear model of the ball and plate system that was found by applying Lagrange's method, the following section illustrates the steps followed.

According to Lagrange's method, the model that describes the dynamics of the system can be found from the following equation :

$$
\frac{\partial}{\partial t}\left(\frac{\partial L}{\partial \dot{x}}\right)-\frac{\partial L}{\partial x}=0
$$

With $\mathrm{L}$ is the difference between the kinetic energy and the potential energy of the ball:

$$
L=E_{k}-E_{p}
$$

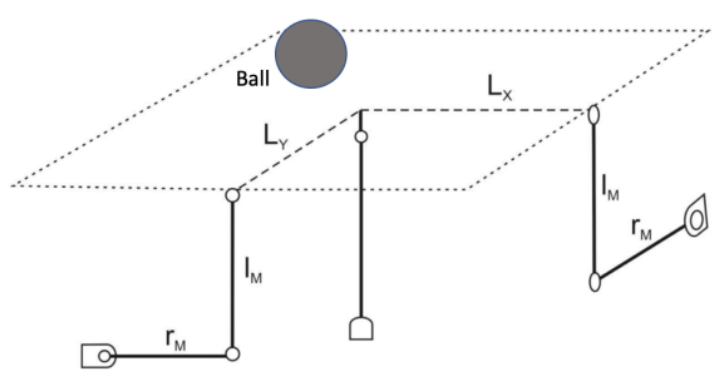


Fig.1. Ball-plate system structure

Neglecting slippage, $E_{k}$ can be written as :

$$
E_{k}=\frac{1}{2} m_{b}\left(\dot{x}_{b}^{2}+\dot{y}_{b}^{2}\right)+\frac{1}{2} J_{b} \omega_{b}^{2}
$$

And $E_{p}$ can be written as :

$$
E_{p}=-m_{b} g x_{b} \sin (\alpha)-m_{b} g y_{b} \sin (\beta)
$$

Based on equations (1),(2),(3) and (4) we find the equation that governs the dynamics of the ball along the $\mathrm{x}$-axis:

$$
\ddot{x}_{b}=\frac{m_{b} g r_{b}^{2}}{m_{b} r_{b}^{2}+J_{b}} \sin (\alpha)
$$

The equation (5) represents the evolution of the ball along the $\mathrm{x}$ axis as a function of the angle $\alpha$.

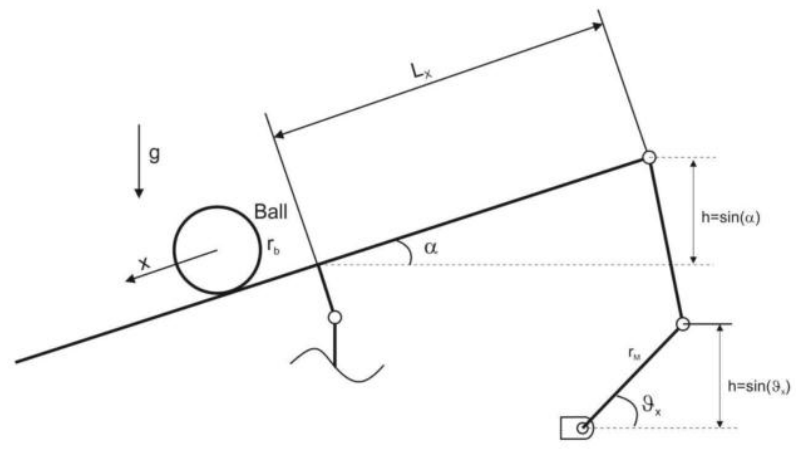

Fig.2. The ball-plate system in side view

In our case, the controller acts on the angle $\vartheta_{x}$, thus using the following equation :

$$
\sin \left(\vartheta_{x}\right) r_{M}=\sin (\alpha) L_{X}=h
$$

We find the equation that links the input of the system $\vartheta_{x}$ and the output of the system $x_{b}$ :

$$
\ddot{x}_{b}=\frac{m_{b} g r_{b}^{2} r_{M}}{\left(m_{b} r_{b}^{2}+J_{b}\right) L_{X}} \sin \left(\vartheta_{x}\right)
$$

Similarly, following the same approach, the equation that describes the evolution of the ball along the $y$-axis is as follows:

$$
\ddot{y}_{b}=\frac{m_{b} g r_{b}^{2} r_{M}}{\left(m_{b} r_{b}^{2}+J_{b}\right) L_{Y}} \sin \left(\vartheta_{y}\right)
$$


Under the hypothesis of small variation of $\vartheta_{x}$ and $\vartheta_{y}$, equations (7) and (8) become :

$$
\begin{aligned}
\ddot{x}_{b} & =G_{x} \vartheta_{x} \\
\ddot{y}_{b} & =G_{y} \vartheta_{y}
\end{aligned}
$$

with

$$
G_{x}=\frac{m_{b} g r_{b}^{2} r_{M}}{\left(m_{b} r_{b}^{2}+J_{b}\right) L_{X}} \text { and } G_{y}=\frac{m_{b} g r_{b}^{2} r_{M}}{\left(m_{b} r_{b}^{2}+J_{b}\right) L_{Y}}
$$

Remark: Because of the similarity of the models along the $\mathrm{x}$-axis and the $\mathrm{y}$-axis, in the following we will perform the study of the observer-based state feedback controller along the $\mathrm{x}$-axis, and deduce the results for the y-axis.

Thus, taking into consideration the feedback delay $\tau_{1 x}$, the state-space model along the $\mathrm{x}$-axis is defined by:

$$
\left\{\begin{array}{l}
\dot{x}(t)=A x(t)+B u\left(t-\tau_{1 x}\right) \\
y(t)=C x(t)
\end{array}\right.
$$

Where $x(t)=\left[\begin{array}{ll}x_{b}(t) & \dot{x}_{b}(t)\end{array}\right]^{T}, u(t)=\vartheta_{x}(t)$

and

$$
A=\left[\begin{array}{ll}
0 & 1 \\
0 & 0
\end{array}\right], \quad B=\left[\begin{array}{c}
0 \\
G_{x}
\end{array}\right], \quad C=\left[\begin{array}{ll}
1 & 0
\end{array}\right]
$$

\section{Observer-based state feedback control}

\section{A. The Luenberger Observer}

We will now present the principal objective of this paper, which is to set up a real-time observer of the state of system $x(t)$. Equation (12) defines the structure of the observer that will be applied, where $L_{x}=\left[\begin{array}{ll}l_{x 1} & l_{x 2}\end{array}\right]^{T}$ is the gain of the observer that will allow the estimation error $e(t)=x(t)-\hat{x}(t)$ to converge to zero. And $\tau_{2 x}$ is the delay introduced by the Luenberger Observer into the measurement loop.

$$
\left\{\begin{aligned}
\dot{\hat{x}}(t)= & A \hat{x}(t)+B u\left(t-\tau_{1 x}\right) \\
& +L_{x}\left[y\left(t-\tau_{2 x}\right)-\hat{y}\left(t-\tau_{2 x}\right)\right] \\
\hat{y}(t)= & C \hat{x}(t)
\end{aligned}\right.
$$

Applying the Laplace transform, equation (12) will become :

$$
\left\{\begin{aligned}
s \hat{x}(s)= & A \hat{x}(s)+B u(s) e^{-\tau_{1 x} s} \\
& +L_{x}[y(s)-\hat{y}(s)] e^{-\tau_{2 x} s} \\
\hat{y}(s)= & C \hat{x}(s)
\end{aligned}\right.
$$

Considering the state feedback controller defined by : 


$$
u(t)=-K_{x} \hat{x}(t)
$$

We find the equation that governs the dynamics of the estimation error :

$$
s e(s)=A e(s)-L_{x} C e(s) e^{-\tau_{2 x} s}
$$

And the characteristic equation is :

$$
H\left(s, e^{-\tau_{2 x} s}\right)=\operatorname{det}\left(s I_{2}-\left(A-L_{x} C e^{-\tau_{2 x} s}\right)\right)=0
$$

After simplification, the characteristic equation can be written as :

$$
H\left(s, l_{x 1}, l_{x 2}, \tau_{2 x}\right)=Q_{L}(s)+P_{L}(s) e^{-\tau_{2 x} s}
$$

With

$$
Q_{L}(s)=s^{2}, \quad P_{L}(s)=s l_{x 1}+l_{x 2}
$$

\section{B. Routh-Hurwitz Criterion}

The Routh-Hurwitz Criterion allows analysis of the system's stability. In our case, we will use this criterion to determine the conditions that guarantee the stability based on the Luenberger Observer. To do this, let us first consider a zero delay $\tau_{2 x}=0$, the equation (17) becomes :

$$
H\left(s, l_{x 1}, l_{x 2}, 0\right)=s^{2}+s l_{x 1}+l_{x 2}
$$

So, by applying Routh's criterion to this polynomial, we have found that the parameters of the observer's gain $L_{x}$ must be strictly positive :

$$
l_{x 1}>0, \quad l_{x 2}>0
$$

\section{Frequency Domain Analysis}

In this section, we will make a more general study of the system stability. To do so, we will first consider a non-zero delay $\tau_{2 x}$, then we will consider that equation (17) has a pure imaginary root, i.e. the system is at the limit of stability, so we have :

$$
\forall \omega>0, \exists \tau_{2 x}^{*} \in \mathcal{R}^{+}, \quad H\left(j \omega, l_{x 1}, l_{x 2}, \tau_{2 x}^{*}\right)=0
$$

Considering the real and imaginary part, we find:

$$
\left\{\begin{array}{r}
-\omega^{2}+\omega l_{x 1} \sin \left(\omega \tau_{2 x}^{*}\right)+l_{x 2} \cos \left(\omega \tau_{2 x}^{*}\right)=0 \\
\omega l_{x 1} \cos \left(\omega \tau_{2 x}^{*}\right)-l_{x 2} \sin \left(\omega \tau_{2 x}^{*}\right)=0
\end{array}\right.
$$

i.e. : 


$$
\begin{gathered}
l_{x 1}=\omega \sin \left(\omega \tau_{2 x}^{*}\right) \\
l_{x 2}=\omega^{2} \cos \left(\omega \tau_{2 x}^{*}\right)
\end{gathered}
$$

For each value of $\omega$, the equations (21) and (22) give us a pair $\left(l_{x 1}, l_{x 2}\right)$ that places the system at the limit of stability, so by varying $\omega$ we find a curve that represents a limit of the regions of stability of the system.

Now we are going to determine the direction of passage [7] given by the sign of $R_{2} I_{1}-$ $R_{1} I_{2}$, with the real and imaginary parts $R_{i}$ and $I_{i}$ are defined by:

$$
\begin{aligned}
& R_{1}+j I_{1}=-\left.\frac{1}{s} \frac{\partial H\left(s, l_{x 1}, l_{x 2}, \tau_{2 x}^{*}\right)}{\partial l_{x 2}}\right|_{s=j \omega} \\
& R_{2}+j I_{2}=-\left.\frac{1}{s} \frac{\partial H\left(s, l_{x 1}, l_{x 2}, \tau_{2 x}^{*}\right)}{\partial l_{x 1}}\right|_{s=j \omega}
\end{aligned}
$$

After finding $R_{i}$ and $I_{i}$, we have :

$$
R_{2} I_{1}-R_{1} I_{2}=-\frac{1}{\omega}<0
$$

This means that when $\omega$ varies in the direction of positive values a solution of (20) crosses the imaginary axis from right to left. Thus, using the results found (19), (21), (22) and (23), we have identified the region of stability of the closed-loop system. Fig. 3 shows this region in the case $\tau_{2 x}^{*}=0.4 \mathrm{~s}$

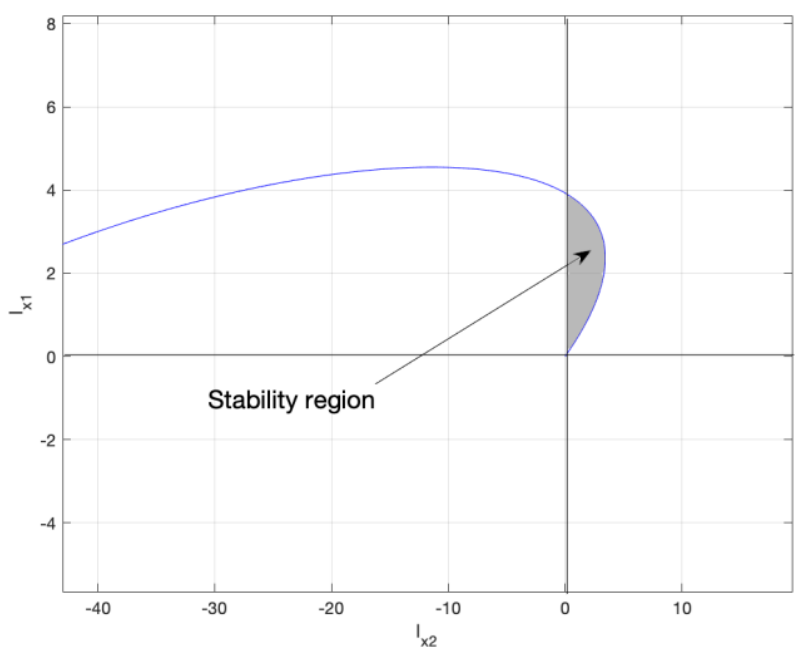

Fig.3. Stability region in $\left(l_{x 2}, l_{x 1}\right)$ plane with $\tau_{2 x}^{*}=0.4$

Every point $\left(l_{x 1}^{*}, l_{x 2}^{*}\right)$ belonging to the region of stability has defined an observation 
gain $L_{x}^{*}=\left[\begin{array}{ll}l_{x 1}^{*} & l_{x 2}^{*}\end{array}\right]^{T}$ where the characteristic equation $H\left(s, l_{x 1}^{*}, l_{x 2}^{*}, \tau_{2 x}\right)=0$ is Hurwitz for any delay $\tau_{2 x}<\tau_{2 x}^{*}$, where $\tau_{2 x}^{*}$ is the critical delay.

\section{Critical Delay}

The stability of the closed-loop system requires that all the roots of the characteristic equation (17) have a strictly negative real part. Thus, to determine the critical delay $\tau_{2 x}^{*}$, we will consider the limit of stability which corresponds to a pure imaginary root of equation (17), i.e. :

$$
Q_{L}(j \omega)+P_{L}(j \omega) e^{-\tau_{2 x} j \omega}=0
$$

Thus, we have :

$$
\cos \left(\omega \tau_{2 x}^{*}\right)-\mathrm{j} \sin \left(\omega \tau_{2 x}^{*}\right)=\frac{\omega^{2}}{j \omega l_{x 1}^{*}+l_{x 2}^{*}}
$$

Thus, the critical delay is :

$$
\tau_{2 x}^{*}=\frac{1}{\omega} \operatorname{Arccos}\left[\frac{l_{x 2}^{*} \omega^{2}}{\left(l_{x 2}^{*}\right)^{2}+\left(\omega l_{x 1}^{*}\right)^{2}}\right]
$$

\section{E. Luenberger Observer along the y-axis}

The Luenberger observer allowing the estimation of the system state along the y-axis is characterized by the observation gain $L_{y}=\left[\begin{array}{ll}l_{y 1} & l_{y 2}\end{array}\right]^{T}$. Following the same process as described in the previous sections, we identified the region of stability of the closedloop system according to the y-axis. Fig. 4 shows this region in the case $\tau_{2 y}^{*}=1 \mathrm{~s}$

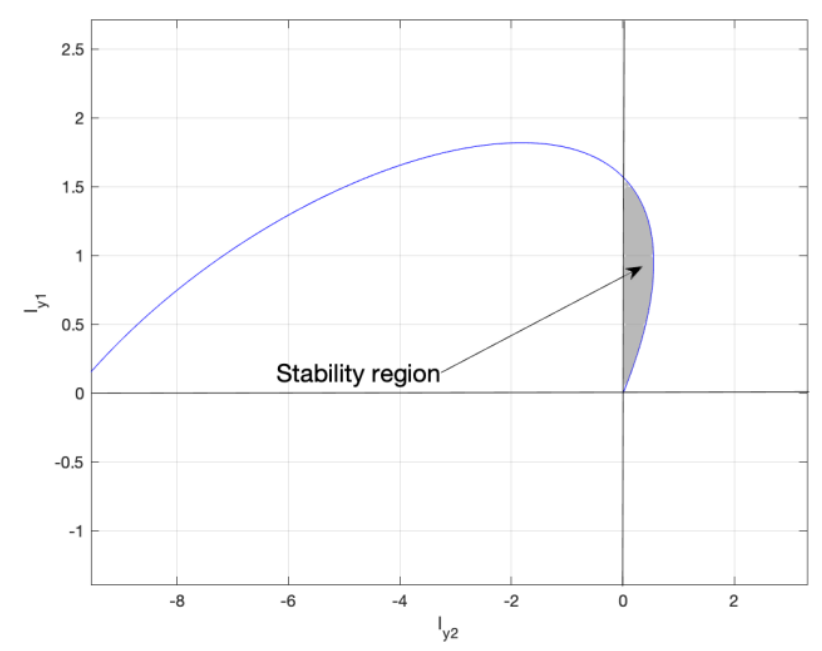

Fig.4. Stability region in $\left(l_{y 2}, l_{y 1}\right)$ plane with $\tau_{2 y}^{*}=1$

Also the critical delay $\boldsymbol{\tau}_{\mathbf{2 y}}^{*}$ is defined by : 


$$
\tau_{2 y}^{*}=\frac{1}{\omega} \operatorname{Arccos}\left[\frac{l_{y 2}^{*} \omega^{2}}{\left(l_{y 2}^{*}\right)^{2}+\left(\omega l_{y 1}^{*}\right)^{2}}\right]
$$

\section{SIMULATION AND RESULTS}

To test the results found, let's consider the ball-plate system with the following parameters : $L_{X}=0.134 \mathrm{~m}, L_{Y}=0.168 \mathrm{~m}, r_{M}=0.0245 \mathrm{~m}, r_{b}=0.02 \mathrm{~m}, J_{b}=$ $0.0000416 \mathrm{~kg} * \mathrm{~m}^{2}, m_{b}=0.26 \mathrm{~kg}, \tau_{2 x}=0.4 \mathrm{~s}, \tau_{2 y}=1 \mathrm{~s}$.

Our objective is to control the position of the ball on the plate, which means that the observer's estimation errors tend towards zero. In order to verify this result, we will choose different gain $L_{x}=\left[\begin{array}{ll}l_{x 1} & l_{x 2}\end{array}\right]^{T}$. and $L_{y}=\left[\begin{array}{ll}l_{y 1} & l_{y 2}\end{array}\right]^{T}$ in the regions shown in Fig.3 and Fig.4 respectively, and then, using equations (24) and (25), we will calculate the critical delays. The obtained results are presented in Table I and Table II.

TABLE I. Selected gains for the closed loop in the $\mathrm{x}$-axis

\begin{tabular}{|c|c|c|}
\hline$\left(l_{x 1}^{*}, l_{x 2}^{*}\right)$ & $L_{x}^{*}$ & $\tau_{2 x}^{*}$ \\
\hline$(3.35,0.69)$ & $\mathrm{L}_{1}=\left[\begin{array}{ll}3.35 & 0.69\end{array}\right]^{\mathrm{T}}$ & 0.4498 \\
\hline$(2.43,2.54)$ & $\mathrm{L}_{2}=\left[\begin{array}{ll}2.43 & 2.54\end{array}\right]^{\mathrm{T}}$ & 0.4551 \\
\hline$(1.44,1.23)$ & $\mathrm{L}_{3}=\left[\begin{array}{ll}1.44 & 1.23\end{array}\right]^{\mathrm{T}}$ & 0.6684 \\
\hline
\end{tabular}

TABLE II. Selected gains for the closed loop in the y-axis

\begin{tabular}{|c|c|c|}
\hline$\left(l_{y 1}^{*}, l_{y 2}^{*}\right)$ & $L_{y}^{*}$ & $\tau_{2 y}^{*}$ \\
\hline$(1.51,0.09)$ & $\mathrm{L}_{4}=\left[\begin{array}{ll}1.51 & 0.09\end{array}\right]^{\mathrm{T}}$ & 1.0134 \\
\hline$(1.05,0.36)$ & $\mathrm{L}_{5}=\left[\begin{array}{ll}1.05 & 0.36\end{array}\right]^{\mathrm{T}}$ & 1.1535 \\
\hline$(0.53,0.25)$ & $\mathrm{L}_{6}=\left[\begin{array}{ll}0.53 & 0.25\end{array}\right]^{\mathrm{T}}$ & 1.4468 \\
\hline
\end{tabular}

By considering the gains $L_{x}^{*}, L_{y}^{*}$ shown in Table I and Table II, and the state feedback gains $K_{x}=\left[\begin{array}{ll}0.45 & 0.6\end{array}\right], K_{y}=\left[\begin{array}{ll}0.35 & 0.75\end{array}\right]$ developed in [1], we obtained the time evolutions of the ball position shown in Fig. 5 and Fig. 6, and the time evolutions of the observation errors $e_{1}(t)=x_{b}(t)-\widehat{x_{b}}(t)$ and $e_{2}(t)=\dot{x}_{b}(t)-\widehat{\dot{x}_{b}}(t)$ shown in Fig. 7, Fig. 8, Fig. 9 and Fig. 10. 


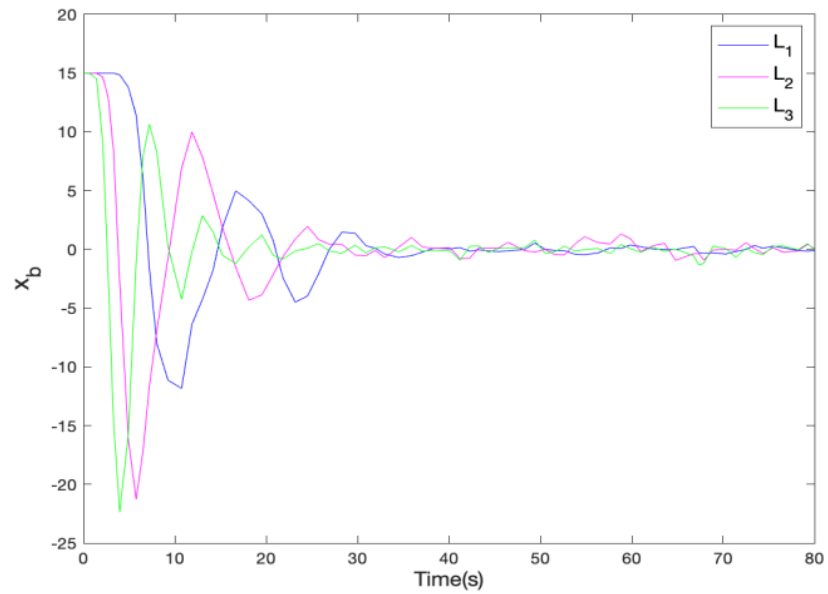

Fig.5. Temporal evolution of $x_{b}$ for the observer gains $L_{1}, L_{2}$ and $L_{3}$

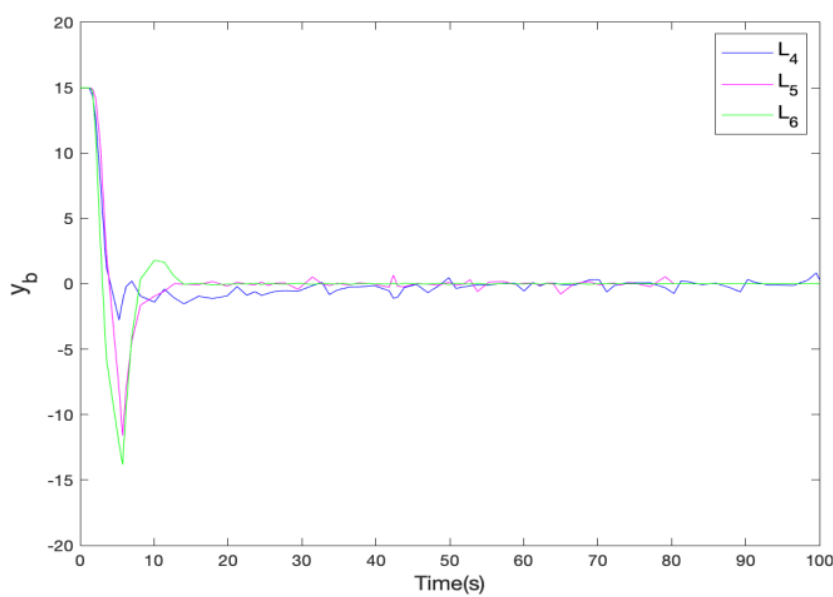

Fig.6. Temporal evolution of $y_{b}$ for the observer gains $L_{4}, L_{5}$ and $L_{6}$

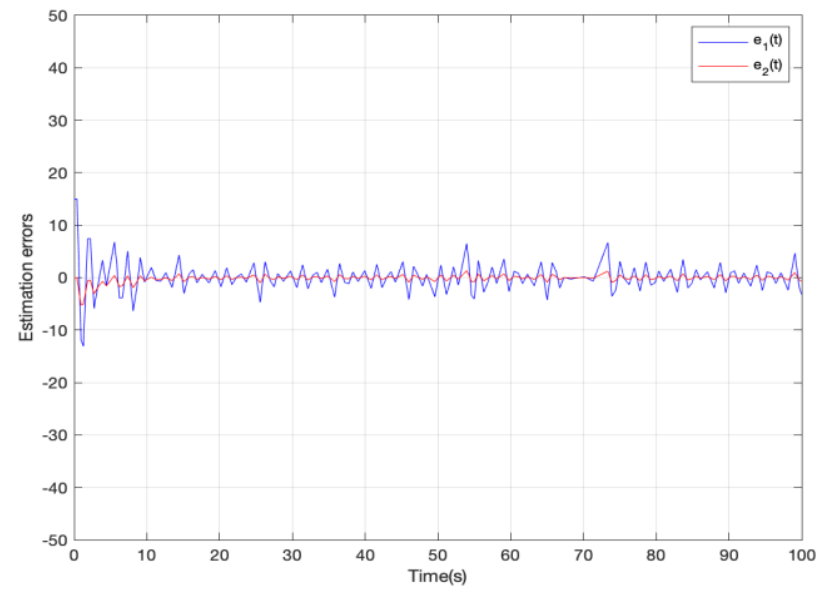

Fig.7. Evolution of observer errors for the gain $L_{1}$ 


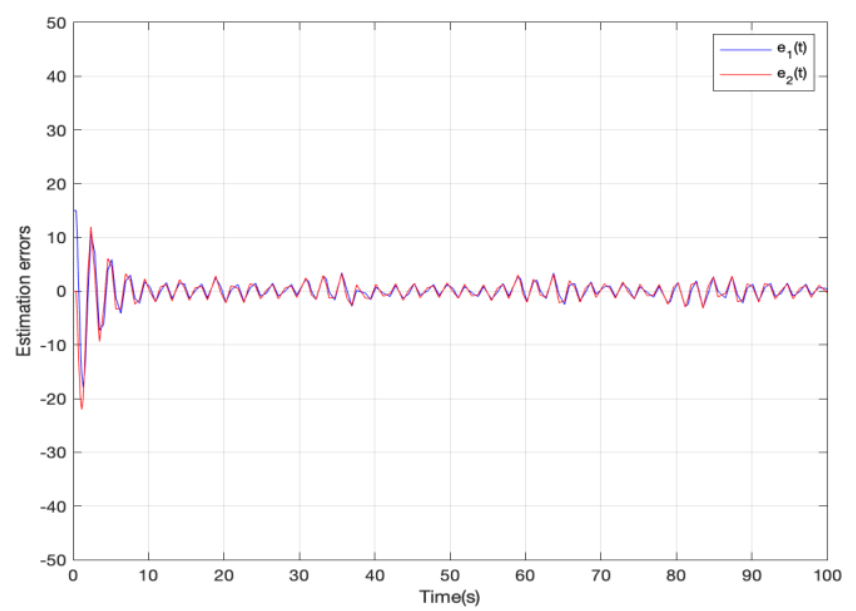

Fig.8. Evolution of observer errors for the gain $L_{2}$

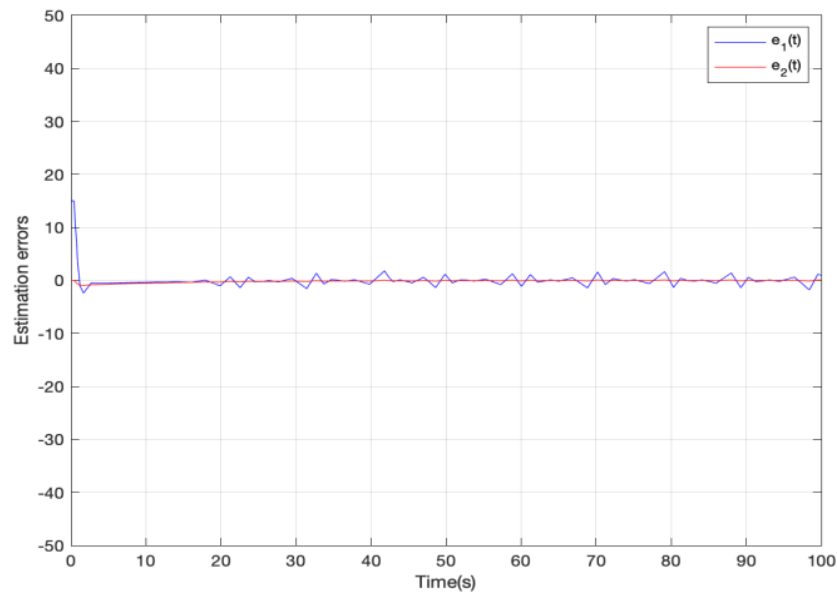

Fig.9. Evolution of observer errors for the gain $L_{4}$

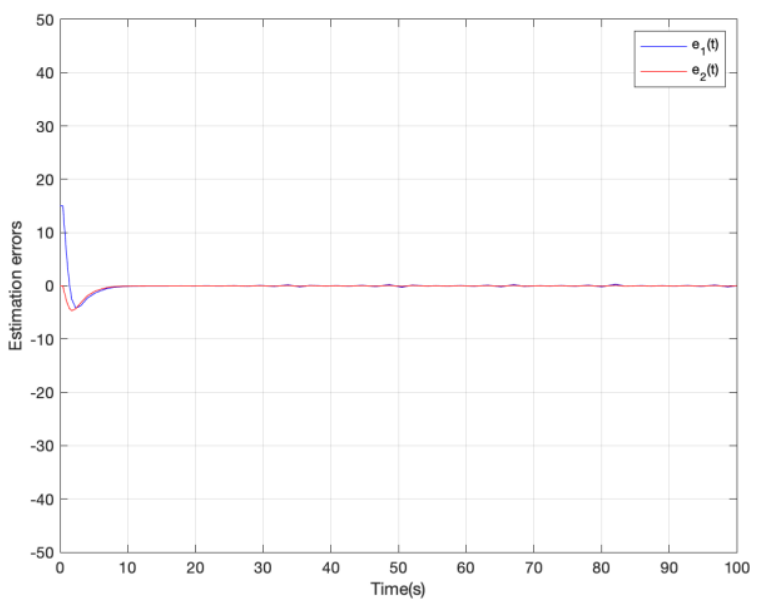

Fig.10. Evolution of observer errors for the gain $L_{5}$

Fig. 7, Fig. 8, Fig. 9 and Fig. 10 show that all the gains of observers, selected in the 
region of stability, allow a zero estimation error, and therefore achieve the controller's objective, i.e. $\left(x_{b}, y_{b}\right)=(0,0)$ (see Fig. 5 and Fig. 6). However, we also find that, according to the selected gain, the dynamic behaviour changes. Thus, for the evolution of the ball along the $\mathrm{x}$-axis, the gain that offers the best response time is $\mathrm{L}_{3}$ (see Fig. 5). For the $y$-axis evolution, $\mathrm{L}_{5}$ offers the best response time (see Fig. 6). However, if the aim is to have a low first overshoot, then the gain $\mathrm{L}_{4}$ should be chosen (see Fig. 5).

We note also that for the selected gains (see Table I and Table II), it is gains $\mathrm{L}_{3}$ and $\mathrm{L}_{6}$ that tolerate respectively higher delays $\tau_{2 x}$ and $\tau_{2 y}$ i.e. the convergence of the ball to the $\left(x_{b}, y_{b}\right)=(0,0)$ coordinates will be ensured for any delay $\tau_{2 x}$ belonging to [0, $0.6684]$ and any delay $\tau_{2 y}$ belonging to $[0,1.4468]$.

\section{CONCLUSION}

In this paper, the problem of the control of the ball-plate system was studied using a frequency domain approach. The developed controller is based, on the one hand, on a state feedback controller and, on the other hand, on the Luenberger observer which allows to estimate the state of the system, thus allowing to increase the controller performance. Simulation results have shown the effectiveness of our approach, however, we find that the choice of observer gains within the stability regions is difficult. Thus, we propose as future work to develop an algorithm that will allow, by taking into account the requirements of the specifications, to choose the best observer gains.

\section{REFERENCES}

[1] A. Gustavo \& L. Juan \& J. B. Jovani, "Control of a ball-and-plate system using a State-feedback controller". Ingeniare. 28. 6-15. 10.4067/S071833052020000100006, 2020.

[2] N. Kosuke, I. Masashi, “A noncontact permanent magnet levitation table with electromagnetic control and it's vibration isolation method using direct disturbance cancellation combining optimal regulators", IEEE Tran On Magnetics, 31 (1), 1995

[3] W. Yu, "Nonlinear PD Regulation for Ball and Beam System", International Journal of Electrical Engineering Education, Vol. 46, pp. 37-59, 2009.

[4] F. Salem, "Mechatronics Design of Ball and Beam System: Education and Research", Control Theory and Informatics 3, 1-26, 2013.

[5] M. Yaseen, J. A. Haider, "Modeling and control for a magnetic levitation system based on SIMLAB platform in real time", Results in Physics, Volume 8, Pages 153-159, ISSN 2211-3797, 2018.

[6] C. E. Lin, C. C. Ker, R. T. Wang and C. L. Chen, "A new ball and beam system using magnetic suspension actuator", 31st Annual Conference of IEEE Industrial Electronics Society. IECON 2005, Raleigh, NC, 2005. 
[7] K. Lefrouni, R. Ellaia, "Congestion control in tcp network with observer-based state-feedback and geometric considerations", Applied Mathematical Sciences, 9(100) :4987- 4996, 2015.

[8] R. Soni, S. Sathans, "Optimal control of a ball and beam system through LQR and LQG", 2nd International Conference on Inventive Systems and Control (ICISC), Coimbatore, pp. 179-184, 2018.

[9] H. Liu, Y. Liang, "Trajectory tracking sliding mode control of ball and plate system", 2nd International Asia Conference on Informatics in Control, Automation and Robotics (CAR 2010), pp. 142-145, Wuhan, 2010.

[10] A. Kassem, H. Haddad, C. Albitar, "Comparison Between Different Methods of Control of Ball and Plate System with 6DOF Stewart Platform", IFACPapersOnLine, Volume 48, Issue 11, Pages 47-52, ISSN 2405-8963, 2015.

[11] D. Meiling, L. Bingyou, L. Wang, "Position control for ball and beam system based on active disturbance rejection control", Systems Science \& Control Engineering. 7. 97-108. 10.1080/21642583.2019.1575297, 2019.

[12] E. Fabregas, J. Chacón, S. Dormido-Canto, G. Farias, S. Dormido, "Virtual Laboratory of the Ball and Plate System", IFAC-Papers On Line, Volume 48, Issue 29, Pages 152-157, ISSN 2405-8963, 2015.

[13] S. K. Valluru, M. Singh, S. Singh, "Prototype design and analysis of controllers for one dimensional ball and beam system”, IEEE 1st International Conference on Power Electronics, Intelligent Control and Energy Systems (ICPEICES), Delhi, pp. 1-6, 2016. 